\section{GAS PHASE INTERSTELLAR CHEMISTRY AND MICROWAVE SPECTROSCOPY OF $\mathrm{CBrClF}_{2}$}

\section{R P A Bettens \\ Department of Chemistry, Monash University, Clayton Vic 3168 January 1992}

The interstellar chemistry of dark molecular clouds is reviewed. Models are proposed to explain the observed strong correlation between infrared and radio fluxes for diverse types of disc galaxies, and to explain the $\mathrm{NH}_{3}$ synthesis problem. The influence of efficient neutral-neutral atomic Oxygen reactions on the formation of complex molecules is discussed.

Separately, the microwave spectrum of the terrestrial atmospheric pollutant $\mathrm{BCF}$ is predicted theoretically and obtained experimentally for the first time.

\section{DARK MATTER IN SPIRAL GALAXIES}

\author{
Markus Buchhorn \\ Mount Stromlo and Siding Spring Observatories, Australian \\ National University, Canberra ACT 2601 \\ April 1992
}

A sample of galaxies drawn from the Mathewson, Ford and Buchhorn (1992) survey is analysed to determine global trends in dark matter halo parameters with galactic parameters. Of the original 1355 galaxies, 582 galaxies are analysed to greater detail. Each galaxy has full 2D $I$ band CCD photometry as well as a major axis rotation curve derived from $H_{a}$ emission. A further 12 galaxies also have $21 \mathrm{~cm}$ measurements yielding rotation curves beyond the edge of the optical disk. This sample is an order of magnitude larger than what is currently in the literature.

For each galaxy, the global parameters Total Luminosity, Central Surface Brightness, Scalelength, Diameter and Morphological Type are derived. The surface brightness distributed is used to calculate the expected rotation curve, assuming a constant $M / L$ with radius. These models are fitted to the data using a variety of approaches, each yielding a value for the luminous $M / L$ ratio. There is general agreement between the various approaches. The Strict Maximum Disk hypothesis appears to be valid, indeed it often provides the only acceptable fit, at least for the brightest/largest galaxies; for fainter galaxies the evidence is weak.

Using the disk $M / L$ value, a pseudo-isothermal halo is fitted to the residuals, yielding halo parameters: central density, core radius and total mass (to some finite radius). The correlations between the halo parameters and the galactic parameters are measured. The CSB appears to be generally independent of the halo parameters. while the scalelength of the disk is strongly correlated with the core radius of the halo with a slope of 1 .

The halo parameters vary strongly with global parameters such as luminosity and diameter. The central density increases as one moves towards fainter, smaller galaxies, while the core radius becomes shorter in these systems. The total halo mass increases linearly with luminosity, but the dominance of the halo over the disk varies in the opposite sense. Smaller, fainter galaxies are much more dominated than the brighter galaxies. In this sample the halo/disk ratio typically varied from around 0.5 to 5 .
The trends found are extrapolated to galaxies outside the sample, and compared with published data for dwarf galaxies in particular. There is general agreement between the expected and the derived halo parameters, although I find that the trends predict higher densities and shorter core radii than are actually observed. This may be due to errors in the determination of the intercept of these trends, or real errors in the analysis of the dwarf galaxy systems.

The global $M / L$ ratio, i.e. total mass divided by total light, is derived for all of the galaxies in the sample, and has a surprisingly narrow distribution. The evidence from analysis of clusters suggests that the global mass-to-light ratio is in fact a constant. This constancy, together with the luminositydiameter correlation yields the well known Tully-Fisher relation. It may actually provide a more reliable distance indicator, since it apears to behave more consistently than the TF, with more uniform slopes and smaller dispersions. In terms of distance errors it unfortunately does not provide an improvement.

The constancy further explains a strong correlation between the halo/disk mass ratio and the luminous $M / L$ ratio. It appears that the star formation in the disk is strongly dependent on the halo mass, or the star formation procedure actually determines the halo mass. This is somewhat easier to understand if the halo is baryonic, but non-baryonic is still certainly possible.

A similar analysis is carried out for stellar systems outside the sample here, using published data. For elliptical galaxies there appears to be a strong relation, but the power law has an unexpected slope. This turns out to be due to the use of an inappropriate diameter, a different diameter produces excellent agreement. For dwarf galaxies the result agrees in general with the spiral galaxies. For globular clusters there is also a very strong relation, possibly very similar to the dwarf galaxy systems in slope and intercept. In all cases (elliptical galaxies, spiral galaxies, dwarf galaxies and globular clusters) the relations are tight enough to be used as a distance indicator.

These results suggest that all types of stellar systems covering a range of over 8 orders of magnitude have very similar origins; it is unfortunately not possible at this stage to determine how the global mass-to-light ratio varies from the smallest systems to the largest.

Overall it appears that the global parameters (Total Luminosity, Diameter, Linewidth) of the system are the most important, yielding the strongest correlations. The secondary parameters such as CSB and scalelength are perhaps only the results of the galaxy's evolutionary history.

\section{DUST OPACITY AND STRUCTURE OF SPIRAL GALAXIES}

\section{Yong-lk Byun}

Mount Stromlo and Siding Spring Observatories, Australian

National University, Canberra ACT 2601

August 1992

This thesis presents a series of theoretical and observational studies on the problem of internal extinction in spiral galaxies. First, in order to understand the effect of absorption and scattering, we perform detailed radiative transfer simulations for a range of model galaxies. This is the first numerical study of this kind, where both absorption and scattering are considered for a three-dimensional distribution of stars 